\title{
Jasmonate signaling is activated in the very early stages of iron deficiency responses in rice roots
}

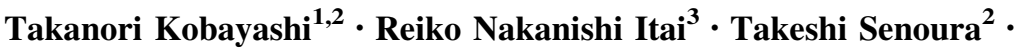 \\ Takaya Oikawa $^{4} \cdot$ Yasuhiro Ishimaru $^{4} \cdot$ Minoru Ueda $^{4} \cdot$ Hiromi Nakanishi $^{3}$. \\ Naoko K. Nishizawa ${ }^{2}$
}

Received: 7 October 2015/ Accepted: 23 April 2016/Published online: 3 May 2016

(C) The Author(s) 2016. This article is published with open access at Springerlink.com

\begin{abstract}
Under low iron availability, plants induce the expression of various genes involved in iron uptake and translocation at the transcriptional level. This iron deficiency response is affected by various plant hormones, but the roles of jasmonates in this response are not well-known. We investigated the involvement of jasmonates in rice iron deficiency responses. High rates of jasmonate-inducible genes were induced during the very early stages of iron deficiency treatment in rice roots. Many jasmonate-inducible genes were also negatively regulated by the ubiquitin ligases OsHRZ1 and OsHRZ2 and positively regulated by the transcription factor IDEF1. Ten out of 35 genes involved in jasmonate biosynthesis and signaling were rapidly induced at $3 \mathrm{~h}$ of iron deficiency treatment, and this induction preceded that of known iron deficiencyinducible genes involved in iron uptake and translocation. Twelve genes involved in jasmonate biosynthesis and signaling were also upregulated in HRZ-knockdown roots.
\end{abstract}

Electronic supplementary material The online version of this article (doi:10.1007/s11103-016-0486-3) contains supplementary material, which is available to authorized users.

Takanori Kobayashi

abkoba@ishikawa-pu.ac.jp

1 Japan Science and Technology Agency, PRESTO, 4-1-8

Honcho, Kawaguchi, Saitama 332-0012, Japan

2 Research Institute for Bioresources and Biotechnology, Ishikawa Prefectural University, 1-308 Suematsu, Nonoichi, Ishikawa 921-8836, Japan

3 Graduate School of Agricultural and Life Sciences, The University of Tokyo, 1-1-1 Yayoi, Bunkyo-ku, Tokyo 113-8657, Japan

4 Graduate School of Science, Tohoku University, 6-3 Aramaki-aza Aoba, Aoba-ku, Sendai 980-8578, Japan
Endogenous concentrations of jasmonic acid and jasmonoyl isoleucine tended to be rapidly increased in roots in response to iron deficiency treatment, whereas these concentrations were higher in HRZ-knockdown roots under iron-sufficient conditions. Analysis of the jasmonate-deficient cpm 2 mutant revealed that jasmonates repress the expression of many iron deficiency-inducible genes involved in iron uptake and translocation under iron sufficiency, but this repression is partly canceled under an early stage of iron deficiency. These results indicate that jasmonate signaling is activated during the very early stages of iron deficiency, which is partly regulated by IDEF1 and OsHRZs.

Keywords HRZ · IDEF1 - Iron deficiency · Jasmonates · Rice - Ubiquitin ligase

\section{Introduction}

Iron $(\mathrm{Fe})$ is an essential element for virtually all living organisms. Plants take up Fe from the rhizosphere, where it is abundant but only slightly soluble. Fe solubility and thus availability is especially low in calcareous soils, which cover one-third of the world's cultivated areas, severely limiting plant productivity. Therefore, understanding plant responses to low $\mathrm{Fe}$ availability is important for breeding crops with better growth under adverse conditions.

In response to low $\mathrm{Fe}$ availability, plants induce the expression of genes involved in $\mathrm{Fe}$ uptake. This response is categorized into two strategies in higher plants. Nongraminaceous plants utilize the reduction strategy (Strategy I), while graminaceous plants utilize the chelation strategy (Strategy II) (Römheld and Marschner 1986). Strategy I is characterized by induction of ferric-chelate reductase FRO 
at the root surface and subsequent uptake of generated $\mathrm{Fe}^{2+}$ ions by induced IRT transporters (Eide et al. 1996; Robinson et al. 1999; Vert et al. 2002). This $\mathrm{Fe}^{2+}$ uptake is supported by the secretion of protons and phenolic compounds by HA and ABCG/PDR transporters, respectively (Santi and Schmidt 2009; Rodríguez-Celma et al. 2013; Fourcroy et al. 2014; Schmid et al. 2014). Strategy II is mediated by $\mathrm{Fe}(\mathrm{III})$ chelators biosynthesized in graminaceous plants, designated mugineic acid family phytosiderophores (MAs) (Takagi 1976). MAs are secreted to the rhizosphere by TOM1 transporter and chelate Fe(III) (Nozoye et al. 2011). Subsequently, the resulting Fe(III)MAs complexes are absorbed into the roots by YS1/YSL transporters (Curie et al. 2001, 2009; Murata et al. 2006; Inoue et al. 2009). These two strategies have been previously considered mutually exclusive, but some exceptions have been reported recently. For example, rice, a graminaceous plant, biosynthesizes 2'-deoximugineic acid (DMA) among MAs and takes up Fe(III)-DMA as a Strategy II mechanism, but it also takes up $\mathrm{Fe}^{2+}$ by OsIRT1 transporter (Ishimaru et al. 2006). Meanwhile, peanut, a Strategy I plant, also utilizes Fe(III)-MAs when MAs are supplied from the intercropped grasses (Xiong et al. 2013a).

In both strategies, strong induction of the corresponding enzymes and transporters takes place at the transcript level in response to low $\mathrm{Fe}$ availability (Kobayashi and Nishizawa 2012). In Arabidopsis thaliana, a Strategy I plant, this response is primarily mediated by basic helix-loophelix (bHLH) transcription factors FIT and subgroup Ib bHLHs (Colangelo and Guerinot 2004; Jakoby et al. 2004; Yuan et al. 2005, 2008; Sivitz et al. 2012; Wang et al. 2013b). Fe deficiency-inducible gene expression in Arabidopsis is also regulated by PYE and subgroup IVc bHLH transcription factors, and by MYB transcription factors MYB10 and MYB72 (Long et al. 2010; Palmer et al. 2013; Zhang et al. 2015). In rice, the genes involved in $\mathrm{Fe}$ (III)-DMA uptake, $\mathrm{Fe}^{2+}$ uptake and Fe translocation are differentially regulated by various transcription factors (Kobayashi et al. 2014). The genes involved in DMAbased Fe uptake are regulated by the bHLH transcription factors OsIRO2 and OsIRO3, and their upstream transcription factor IDEF1 (Ogo et al. 2007, 2011; Kobayashi et al. 2007, 2009, 2014; Zheng et al. 2010). The Fe ${ }^{2+}$ transporter gene OSIRT1 is regulated by IDEF1 and OsIRO3 (Kobayashi et al. 2007, 2009; Zheng et al. 2010). $\mathrm{Fe}$ translocation within the plant is regulated mainly by the transcription factors IDEF1, IDEF2 and OsbHLH133 (Kobayashi et al. 2007, 2009; Ogo et al. 2008; Wang et al. 2013a). Among the above-mentioned Arabidopsis and rice transcription factors, all but IDEF1, IDEF2 and subgroup IVc bHLHs are transcriptionally induced under $\mathrm{Fe}$ deficiency.
In addition to the specific genes involved in Fe uptake, translocation and their regulation, numerous other genes are transcriptionally induced under Fe-deficient conditions, as characterized by transcriptomic analyses (Schmidt and Buckhout 2011; Kobayashi et al. 2014). In contrast, knowledge of protein-level expression responses under $\mathrm{Fe}$ deficiency remains limited. A recent proteome analysis revealed that the $\mathrm{Fe}$ deficiency responses are conserved only partially between the transcript and protein levels in Arabidopsis (Lan et al. 2011). Protein modification and degradation also play important roles in plant responses to environmental fluctuations. In Arabidopsis, subcellular localization, vacuolar sorting and degradation of $\mathrm{Fe}^{2+}$ transporter IRT1 are controlled by monoubiquitination mediated by a RING ubiquitin ligase IDF1 (Barberon et al. 2011; Shin et al. 2013). The Arabidopsis transcription factor FIT, a central regulator of the Strategy I response, is subjected to degradation via the $26 \mathrm{~S}$ proteasome pathway mediated by unknown E3 ubiquitin ligases (Lingam et al. 2011; Sivitz et al. 2011). This degradation is inhibited by interaction with EIN3 and EIL1, which play central roles in ethylene signaling (Lingam et al. 2011) and is enhanced under Fe-deficient conditions (Sivitz et al. 2011). Two Arabidopsis subgroup IVc bHLH transcription factors, ILR3 and AtbHLH115, are bound to and destabilized by a RING ubiquitin ligase, BTS (Selote et al. 2015). ILR3 and AtbHLH115 also bind to transcription factor PYE (Long et al. 2010). pye knockout mutants are susceptible to $\mathrm{Fe}$ deficiency, whereas bts knockdown mutants are tolerant to Fe deficiency (Long et al. 2010; Zhang et al. 2015), suggesting antagonism between PYE and BTS functions. BTS binds to $\mathrm{Fe}$ and zinc ( $\mathrm{Zn}$ ) (Kobayashi et al. 2013) and is destabilized by $\mathrm{Fe}$ in in vitro translation reactions (Selote et al. 2015), suggesting its role as an intracellular Fe sensor. Rice IDEF1, a central transcriptional regulator of $\mathrm{Fe}$ deficiency responses, also binds to $\mathrm{Fe}^{2+}$ and other divalent metals, and is proposed to also be a Fe sensor (Kobayashi et al. 2012). IDEF1 is subjected to $26 \mathrm{~S}$ proteasome-mediated degradation, which is likely inhibited by interaction with the Bowman-Birk trypsin inhibitor IBP1.1 and possibly by its close homolog IBP1.2 (Zhang et al. 2014). E3 ubiquitin ligases involved in modification or degradation of proteins involved in $\mathrm{Fe}$ deficiency responses in graminaceous plants have not been reported.

Previously, we identified two rice RING ubiquitin ligases, OsHRZ1 and OsHRZ2, which bind to Fe and Zn and negatively regulate $\mathrm{Fe}$ deficiency responses (Kobayashi et al. 2013). OsHRZ1 and OsHRZ2 are close homologs of Arabidopsis BTS and similar proteins are widely present in higher plants and algae (Urzica et al. 2012; Kobayashi et al. 2013), suggesting the conservation of HRZs/BTStype putative Fe sensors in the plant kingdom. Knockdown mutants of OsHRZ1 and OsHRZ2 showed improved 
tolerance to Fe deficiency and accumulation of $\mathrm{Fe}$ and $\mathrm{Zn}$ in shoots and seeds irrespectively of Fe nutritional conditions (Kobayashi et al. 2013). Thus, knockdown of HRZs is a promising method of producing $\mathrm{Fe}$ - and $\mathrm{Zn}$-fortified crops with improved growth under Fe-limiting conditions. Microarray and quantitative RT-PCR analyses showed that expression of the majority of known Fe deficiency-inducible genes involved in Fe uptake and translocation is enhanced in these $H R Z$-knockdown lines, especially under Fe-sufficient conditions. OsHRZ1 and OsHRZ2 possess ubiquitination activity in vitro, but their substrate proteins have not been identified. In addition to the RING Zn-finger domain which mediates ubiquitination, OsHRZ1 and OsHRZ2 possess two additional Zn-finger domains, suggesting potential roles in transcriptional, post-transcriptional or translational regulation.

Various plant hormones and small signaling molecules such as nitric oxide also regulate Fe deficiency responses. Auxin, ethylene and nitric oxide positively regulate $\mathrm{Fe}$ deficiency responses in both graminaceous and non-graminaceous plants (Hindt and Guerinot 2012; Kobayashi and Nishizawa 2012). These three molecules are thought to affect each other and are required for $\mathrm{Fe}$ deficiency responses (García et al. 2011; Hindt and Guerinot 2012). In Arabidopsis, abscisic acid and gibberellin are also suggested to play positive roles in Fe deficiency responses (Lei et al. 2014; Matsuoka et al. 2014), whereas cytokinin and jasmonic acid (JA) act negatively (Séguéla et al. 2008; Maurer et al. 2011). Brassinosteroids negatively regulate the expression of genes involved in Fe uptake and translocation in cucumber and rice (Wang et al. 2012, 2015). Abscisic acid could potentially share the signaling pathway with IDEF1-mediated Fe deficiency responses in rice (Kobayashi et al. 2007, 2009). The roles of gibberellin, cytokinin, and JA in Fe deficiency responses in graminaceous plants remain unclear.

JA and its derivatives, jasmonates (JAs), are oxylipinbased plant hormones rapidly synthesized in response to various stresses, such as wounding and pathogens, subsequently mediating the defense mechanisms against these stimuli (Fonseca et al. 2009; Vleesschauwer et al. 2013). JAs are sensed by the $\mathrm{SCF}^{\mathrm{COI} 1}$ complex of E3 ubiquitin ligase, which targets JAZ repressors for $26 \mathrm{~S}$ proteasomemediated degradation. As a consequence, the bHLH transcription factor MYC2/JIN1 is de-repressed, mediating the expression of JA-regulated genes in the presence of JAs. Studies on the JA receptor COI1 revealed the bioactive component of JA to be jasmonoyl isoleucine (JA-Ile) (Fonseca et al. 2009). Although there is no report on JA function in Fe deficiency responses, with the exception of repressive roles in Arabidopsis (Maurer et al. 2011), recent findings suggest positive involvement of JAs in Fe deficiency responses in rice. Firstly, OsRMC, a JA-induced receptor-like protein, negatively regulates JA-mediated root development (Jiang et al. 2007), and positively regulates $\mathrm{Fe}$ deficiency-inducible genes in rice (Yang et al. 2013). Secondly, two IDEF1-binding Bowman-Birk trypsin inhibitors IBP1.1 and IBP1.2 that putatively mediate Fe deficiency responses via IDEF1 (Zhang et al. 2014) are transcriptionally induced by JA treatment (Yoshii et al. 2010). Lastly, two close homologs of HRZ ubiquitin ligases, LjnsRING in Lotus japonicus and TARF in tobacco, regulate infection by symbiotic bacteria and tobacco mosaic virus, respectively (Shimomura et al. 2006; Yamaji et al. 2010), suggesting possible involvement in JA-mediated defense responses.

In the present study, we investigated whether the JA pathway is involved in rice $\mathrm{Fe}$ deficiency responses. Expression analysis revealed that the JA pathway is rapidly activated during the early stages of Fe deficiency in rice roots, and that this pathway is regulated by OsHRZs and IDEF1. Endogenous concentrations of JAs tended to be increased in rice roots under early Fe deficiency, and were higher in HRZ-knockdown roots under Fe sufficiency. Analysis of a JA-deficiency mutant revealed that JAs regulate the genes involved in Fe uptake and translocation dependent on Fe nutritional status. These results revealed a positive involvement of the JA pathway during the early stages of $\mathrm{Fe}$ deficiency in rice roots.

\section{Materials and methods}

\section{Analysis of the transcriptional relationship between JA and Fe deficiency responses}

The corresponding clones of the JA-responsive genes reported previously by Yoshii et al. (2010) and Seo et al. (2011) were identified on the rice $44 \mathrm{~K}$ microarray slide (Agilent Technologies, USA). Based on the summation of microarray and RNA gel blot analyses of suspension-cultured non-transgenic rice after treatment with $50 \mu \mathrm{M}$ JA for 1 and $6 \mathrm{~h}$ (Yoshii et al. 2010) and microarray analysis of 2-wk-old non-transgenic rice seedlings after treatment with $100 \mu \mathrm{M}$ methyl-JA for $6 \mathrm{~h}$ (Seo et al. 2011), 109 and 29 clones were chosen as early JA-induced and JA-repressed genes, respectively. Based on the microarray analysis of non-transgenic rice after treatment with $30 \mu \mathrm{M}$ JA for 12 days (Yoshii et al. 2010), 287 and 71 clones were chosen as late JA-induced and JA-repressed genes, respectively. Genes involved in JA biosynthesis and signaling were derived from the description by Hirano et al. (2008), except for OsCOI1a and OsCOI1b which were derived from the description by Yang et al. (2012).

Transcriptional early $\mathrm{Fe}$ deficiency responses for the 3-36-h treatments in roots were based on a previous 
microarray analysis by Itai et al. (2013). Transcripts showing $\log _{2}$ expression ratios $>1$ and $<-1$ were grouped as induced and repressed transcripts, respectively, only if they showed a signal value $>20$ and significance by $t$ test within an array slide $(P<0.05)$. Transcriptional responses to 7 days of $\mathrm{Fe}$ deficiency in roots were based on a previous microarray analysis by Ogo et al. (2008). Transcripts with a signal value $>100$ and $P<0.05$ showing $\log _{2}$ expression ratios $>1$ and $<-1$ were grouped as induced and repressed transcripts, respectively. Transcriptional responses to 6 or $24 \mathrm{~h}$ of nitrogen $(\mathrm{N})$, phosphorus $(\mathrm{P})$ or potassium $(\mathrm{K})$ deficiency in roots were based on a previous microarray analysis and threshold (false discovery rate $<0.05$ and fold change $>2.0$ ) described by Takehisa et al. (2013). Transcriptional responses in the HRZ-knockdown roots (which show slight repression of $O S H R Z 1$ expression and stronger repression of $O s H R Z 2$ expression) were evaluated based on Kobayashi et al. (2013). Transcripts with a signal value $>20$ and $P<0.05$ showing average $\log _{2}$ expression ratios for line $2 \mathrm{i}-2 /$ non-transformant and line $2 \mathrm{i}$-3/non-transformant $>1$ were grouped as induced transcripts. Transcriptional responses to $I D E F 1$ induction in roots were based on a previous microarray analysis and threshold described by Kobayashi et al. (2009). Transcripts with a signal value $>100$ and $P<0.05$ showing $\log _{2}$ expression ratios for the IDEFI induction line 13/non-transformant $>1$ were grouped as induced transcripts. Cis-sequence distribution analysis was performed according to Ogo et al. (2008) and Kobayashi et al. (2009). Significant overrepresentation or underrepresentation compared with the average rate for all 42,123 clones was analyzed using a binomial distribution test. Transcriptional responses of $\mathrm{Fe}$ deficiency-related genes to $100 \mu \mathrm{M} \mathrm{JA}$ treatment in roots were derived from the data provided on the RiceXPro website (Sato et al. 2013; http://ricexpro.dna.affrc.go.jp/index.html).

\section{Plant materials and growth conditions}

Non-transgenic rice (Oryza sativa L. cultivar Tsukinohikari) was germinated on Murashige and Skoog medium, while the HRZ-knockdown line 2i-3 (Kobayashi et al. 2013) was germinated on Murashige and Skoog medium with hygromycin B $(50 \mathrm{mg} / \mathrm{L})$. After 18 days of culture followed by 3 days of acclimation, the plantlets were transferred to a hydroponic solution in a greenhouse under a $30{ }^{\circ} \mathrm{C}$ light and $25{ }^{\circ} \mathrm{C}$ dark cycle with natural light conditions. The composition of the hydroponic solution was as follows: $0.70 \mathrm{mM} \mathrm{K}_{2} \mathrm{SO}_{4}, 0.10 \mathrm{mM} \mathrm{KCl}, 0.10 \mathrm{mM}$ $\mathrm{KH}_{2} \mathrm{PO}_{4}, 2.0 \mathrm{mM} \mathrm{Ca}\left(\mathrm{NO}_{3}\right)_{2}, 0.50 \mathrm{mM} \mathrm{MgSO}, 10 \mu \mathrm{M}$ $\mathrm{H}_{3} \mathrm{BO}_{3}, \quad 0.50 \mu \mathrm{M} \quad \mathrm{MnSO}_{4}, \quad 0.50 \mu \mathrm{M} \quad \mathrm{ZnSO}_{4}, \quad 0.20 \mu \mathrm{M}$ $\mathrm{CuSO}_{4}, 0.01 \mu \mathrm{M}\left(\mathrm{NH}_{4}\right)_{6} \mathrm{Mo}_{7} \mathrm{O}_{24}$, and $100 \mu \mathrm{M} \mathrm{Fe}(\mathrm{III})-$ EDTA. The $\mathrm{pH}$ of the nutrient solution was adjusted to 5.5 every $2-3$ days. After 10 days of preculture in hydroponic solution, Fe deficiency was initiated by omitting Fe(III)EDTA from the solution at 10:30 AM. Fe sufficiency (control) plants were cultured similarly with Fe(III)-EDTA. Whole roots were harvested from two plants per replicate at 6 and $24 \mathrm{~h}$ after initiating the treatments, immediately frozen in liquid nitrogen and used for further analyses.

The JA-deficient cpm2 mutant (Riemann et al. 2013) was kindly provided by Dr. Moritoshi Iino (Osaka City University, Japan), Dr. Hisakazu Yamane, Dr. Koji Miyamoto (Teikyo University, Japan) and Dr. Kazunori Okada (The University of Tokyo, Japan). Wild-type rice (cultivar Nihonmasari) and cpm2 mutant were germinated on Murashige and Skoog medium. Homozygous cpm2 mutants were selected by long coleoptile phenotype (Riemann et al. 2013) and confirmed by genomic PCR. Hydroponic culture was carried out as above, except that the preculture in hydroponic solution was shortened to 5 days and Fe-deficiency treatment was extended to 7 days. Relative chlorophyll content of the youngest leaf was measured using a SPAD-502 chlorophyll meter (Konica Minolta, Tokyo, Japan). Hydroponic solution was renewed at 4 days after initiating the treatments. For quantitative RT-PCR analysis, whole roots were harvested at $24 \mathrm{~h}$ after initiating the treatments.

\section{Quantification of endogenous JAs}

Frozen roots (30-200 mg fresh weight) were ground into a powder using a mortar and pestle, and total JAs were extracted with $1 \mathrm{~mL}$ ethanol. After an overnight incubation at $4{ }^{\circ} \mathrm{C}$ in the dark followed by centrifugation at $20,000 \times g$ for $5 \mathrm{~min}, 900 \mu \mathrm{L}$ of the supernatant were transferred to a new tube, and the liquid was evaporated by vacuum at room temperature. The extract was resuspended in $40 \mu \mathrm{L}$ ultrapure water and centrifuged at $20,000 \times g$ for $20 \mathrm{~min}$. A $35-\mu \mathrm{L}$ aliquot was transferred to a new tube and centrifuged again at $20,000 \times g$ for $20 \mathrm{~m}$, after which $30 \mu \mathrm{L}$ were transferred to a 0.8 -mL Sn-Vial (GL-Science, Japan), and $10 \mu \mathrm{L}$ of sample with internal standards were subjected to ultra-performance liquid chromatography coupled to time-of-flight mass spectrometry (UPLC/TOFMS) analysis (Sato et al. 2009). UPLC/TOFMS analysis was performed using an Agilent 1290 Infinity (Agilent Technologies) coupled to a micrOTOF II (Bruker Daltonics, Germany). A ZORBAX Eclipse Plus C18 column (1.8 $\mu \mathrm{m}$, $2.1 \times 50 \mathrm{~mm}$; Agilent Technologies) was used to separate the compounds. The mobile phases were A, $20 \%(\mathrm{v} / \mathrm{v})$ aqueous methanol with $0.05 \%(\mathrm{v} / \mathrm{v})$ acetic acid, and B, methanol with $0.05 \%(\mathrm{v} / \mathrm{v})$ acetic acid. The gradient program was 0-3.5 min, isocratic $90 \% \mathrm{~A} ; 3.5-6$ min, linear gradient $90-0 \% \mathrm{~A} ; 6.1-9 \mathrm{~min}$, isocratic $90 \% \mathrm{~A}$, with a flow rate of $0.15 \mathrm{~mL} / \mathrm{min}$. The mass spectrometer was run in the negative mode with a scan range of $100-700 \mathrm{~m} / \mathrm{z}$. 
The capillary voltage was $4200 \mathrm{~V}$, the nebulizer gas pressure was $1.6 \mathrm{bar}$, the desolvation gas flow was $8.0 \mathrm{~L} /$ min, and the temperature was $180{ }^{\circ} \mathrm{C}$. JA and JA-Ile were quantified on the basis of an extracted ion chromatogram and the corresponding peak position of the standard solution.

\section{Quantitative RT-PCR analysis}

Total RNA was extracted from roots using the RNeasy Plant Mini Kit (Qiagen), treated with DNase I and reversetranscribed using ReverTra Ace qRT-PCR RT Master Mix with gDNA Remover (Toyobo). Real-time PCR was performed in a StepOnePlus ${ }^{\mathrm{TM}}$ Real-Time PCR System (Applied Biosystems) with SYBR Premix Ex $\mathrm{Taq}^{\mathrm{TM}}$ II (TaKaRa) or with TaqMan Gene Expression Assays (Applied Biosystems). The transcript abundance was normalized against the rice $\alpha-2$ tubulin transcript level and was expressed as a ratio relative to the levels in the Fe-sufficient non-transgenic roots at $6 \mathrm{~h}$ or Fe-sufficient wild-type roots. Primers used for quantitative RT-PCR are shown in Online Resource 1.

\section{Results}

\section{JA responses are activated during the very early stages of Fe deficiency}

Using the $44 \mathrm{~K}$ microarray platform, we investigated the expression patterns of genes previously reported to be induced after $1-6 \mathrm{~h}$ or 12 days of JA treatment (Yoshii et al. 2010; Seo et al. 2011). Overlaps of these JA-induced genes with those induced by Fe deficiency were calculated based on our previous microarray studies of early (3-36 h) and subsequent (7 days) Fe deficiency treatments in nontransgenic rice roots (Ogo et al. 2008; Itai et al. 2013) (Fig. 1a). We found notably high overlap of JA-induced genes with the $\mathrm{Fe}$ deficiency-induced genes at the early stages of treatment (Fig. 1a). Among the JA-induced genes at $1-6 \mathrm{~h}, 28 \%$ was also induced at $3 \mathrm{~h}$ of Fe deficiency, and this overlap gradually decreased with the duration of Fe deficiency treatment until $36 \mathrm{~h}$. The overlap of JA-induced genes at 12 days was also the highest at $3 \mathrm{~h}$ of $\mathrm{Fe}$ deficiency $(26 \%)$, but it remained high (13-15\%) throughout the $6-36 \mathrm{~h}$ of $\mathrm{Fe}$ deficiency treatment. Although this rate decreased to $5 \%$ at 7 days of $\mathrm{Fe}$ deficiency, it was still overrepresented in comparison with the total rate of the $\mathrm{Fe}$ deficiency-induced genes at 7 days. These results strongly suggest that the JA responses are rapidly activated at the onset of Fe deficiency.

We also investigated the genes repressed by JA treatment reported by Yoshii et al. (2010) and Seo et al. (2011).

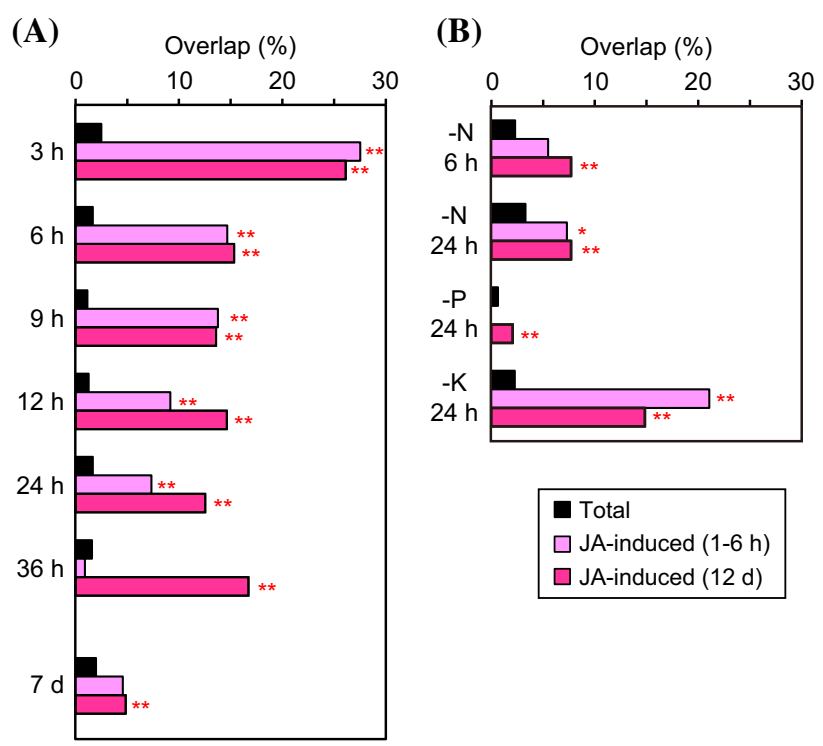

Fig. 1 Overlap of the genes induced by jasmonates (JAs) with the nutrient deficiency-induced genes in roots. a Fe deficiency-induced genes. b N, P or K deficiency-induced genes. Genes induced by JA treatment after $1-6 \mathrm{~h}$ and 12 days (d) were analyzed based on previous reports by Yoshii et al. (2010) and Seo et al. (2011). Fe deficiency-induced genes at 3-36 $\mathrm{h}$ and 7 days are based on previous microarray analyses by Itai et al. (2013) and Ogo et al. (2008), respectively. N, P or K deficiency-induced genes at 6 or $24 \mathrm{~h}$ are based on previous microarray analysis by Takehisa et al. (2013). Overlap was calculated as (the number of the clones showing nutrient deficiency-induction and JA-induction)/(the number of the total clones showing JA-induction). "Total" rate was calculated as (the number of the total clones showing nutrient deficiency-induction)/(the number of the total clones analyzed). Asterisks indicate significant overrepresentations compared with the total rates $(* * P<0.01)$. No significant underrepresentation was observed

The JA-repressed genes at 12 days, but not $1-6 \mathrm{~h}$, constituted significantly high overlap with $\mathrm{Fe}$ deficiency-induced genes, especially during the early stages (Online Resource 2). We also found high overlap of JA-induced and JArepressed genes with Fe deficiency-repressed genes, especially in the combinations of 12-days JA response and 7-days Fe deficiency response (Online Resource 2). These results suggest that both activating and repressive pathways involved in JA responses are linked to both activating and repressive regulation under Fe deficiency.

In order to estimate specificity of the link between JA and $\mathrm{Fe}$ deficiency responses, we also investigated the overlap of JA-induced genes with those induced by other nutrient deficiencies. Based on a previous microarray analysis by Takehisa et al. (2013), N deficiency-induced genes at $6 \mathrm{~h}$ and $\mathrm{N}, \mathrm{P}$ or $\mathrm{K}$ deficiency-induced genes at $24 \mathrm{~h}$ were analyzed (Fig. 1b). Among these, K deficiencyinduced genes showed notably high overlap with JA-induced genes at both $1-6 \mathrm{~h}(21 \%)$ and 12 days (17\%), consistent with previous reports that many $\mathrm{K}$ deficiencyinduced genes are related to JA responses in both rice 
(Takehisa et al. 2013) and Arabidopsis (Armengaud et al. 2004, 2010). N deficiency-induced genes also showed lower but still significant overlap with JA-induced genes at both $1-6 \mathrm{~h}$ and 12 days. $\mathrm{P}$ deficiency-induced genes showed very small overlap. We further investigated the overlap of JA-induced or -repressed genes with N, P or $\mathrm{K}$-induced or -repressed genes (Online Resource 3). N deficiency-induced genes did not show high overlap with JA-repressed genes, while $\mathrm{N}$ deficiency-repressed genes showed significantly overrepresented overlap with JA-induced or -repressed genes. $\mathrm{P}$ deficiency-induced or -repressed genes showed significantly overrepresented overlap with JA-induced or -repressed genes only in limited cases. $\mathrm{K}$ deficiency-induced genes showed large overlap with JA-repressed genes at 12 days, whereas no JA-induced or -repressed genes were repressed by $\mathrm{K}$ deficiency. These results indicate that JA responses are involved in various nutrient deficiencies, and the pattern of the involvement is nutrient-specific, at least for Fe, N, P and $\mathrm{K}$. The high overlap of JA-induced genes at the early stages of deficiency treatment appeared to be characteristic of Fe deficiency. We did not analyze $\mathrm{P}$ or $\mathrm{K}$ deficiencyinduced genes at $6 \mathrm{~h}$, because the numbers of these genes were extremely small (Takehisa et al. 2013).

We then investigated the effects of known regulators of Fe deficiency responses on the expression of JA-induced genes. Based on previous microarray analyses (Kobayashi et al. 2009, 2013), the genes induced in the HRZ-knockdown or IDEF1-induced rice lines, both of which show enhanced Fe deficiency responses (Kobayashi et al. 2007, 2009, 2013), were used for calculating the overlaps with JA-induced genes (Fig. 2a). The genes induced in the HRZknockdown lines under either Fe-sufficient or -deficient conditions showed significantly higher overlap with JAinduced genes at 12 days, but not at 1-6 h (Fig. 2a). In contrast, significant overrepresentation was observed for the IDEF1-induced lines at both 1 and 7 days of Fe deficiency treatments with JA-induced genes at both 1-6 h and 12 days. These results suggest that the late response of the JA pathway is constitutively repressed by OsHRZs, while both the early and late JA responses are activated by IDEF1 under Fe deficiency.

Investigation of cis-acting sequences in JA-responsive gene promoters further revealed a relationship between JAs and Fe deficiency responses (Fig. 2b). JA-induced genes at 12 days, but not at 1-6 h, showed significant overrepresentation of cis-acting elements mediating Fe deficiency responses, namely, core-binding sequences of IDEF1, IDEF2 and OsIRO2 (Kobayashi et al. 2007; Ogo et al. 2006, 2008; Kakei et al. 2013), suggesting the involvement of these elements and transcription factors during late JA responses. JA-induced gene promoters at both $1-6 \mathrm{~h}$ and 12 days also showed overrepresentation of G-box,
(A)

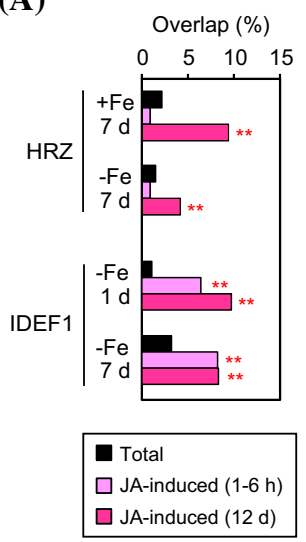

(B)

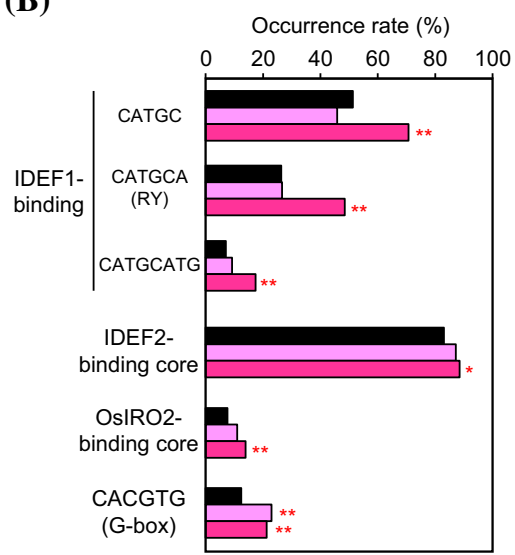

Fig. 2 Involvement of trans-acting regulators and cis-acting elements for $\mathrm{Fe}$ deficiency responses in the expression of genes induced by jasmonates (JAs). a Overlap of the genes induced by JA treatment after $1-6 \mathrm{~h}$ or 12 days (d) with the genes whose expression was induced in HRZ-knockdown (HRZ) or IDEF1-induced (IDEF1) lines under conditions of Fe sufficiency $(+\mathrm{Fe})$ or deficiency $(-\mathrm{Fe})$ after 1 or 7 days in roots based on previous microarray analyses by Kobayashi et al. (2009, 2013). Overlap was calculated as (the number of the clones showing [induction in HRZ-knockdown or $I D E F 1$-induced lines] and JA-induction)/(the number of the total clones showing JA-induction). "Total" rate was calculated as (the number of the total clones showing induction in HRZ-knockdown or $I D E F 1$-induced lines)/(the number of the total clones analyzed). b Occurrence rates of $c i s$-acting sequences in the genes induced by JA treatment after $1-6 \mathrm{~h}$ or 12 days. CATGC, the minimal sequence recognized by IDEF1; CATGCA (RY) and CATGCATG, common binding sequences of IDEF1 and other ABI3/VP1 family transcription factors (Kobayashi et al. 2007); IDEF2-binding core, the minimal sequence recognized by IDEF2 [CA(A/C)G(T/C)(T/C/A)(T/C/A); Ogo et al. 2008]; OsIRO2-binding core, the minimal sequence efficiently recognized by OsIRO2 [CACGTGG; Ogo et al. 2006]; CACGTG (G-box), the binding sequence of many bHLH transcription factors, including MYC2/JIN1, a central regulator of the JA response (Fonseca et al. 2009). The putative promoter regions 500 nucleotides upstream of the $5^{\prime}$ border of the predicted transcription initiation sites were used to search for $c i s$-sequences. Occurrence rate was calculated as (the number of the clones possessing the cis-sequence and showing JA-induction)/(the number of the total clones showing JA-induction). "Total" rate was calculated as (the number of the total clones possessing the $\mathrm{cis}$-sequence)/(the number of the total clones analyzed). Asterisks indicate significant overrepresentations compared with the total rates $(* P<0.05 ; * * P<0.01)$. No significant underrepresentation was observed

presumably reflecting a central JA response mediated by MYC2/JIN1 (Fonseca et al. 2009).

Similar analysis of the JA-repressed genes also revealed overrepresentation of the JA-repressed genes at 12 days, but not at $1-6 \mathrm{~h}$, with the $H R Z$-knockdown lines under Fe sufficiency, the IDEF 1 -induced lines at 7 days of Fe deficiency, and IDEF1-binding cis-acting sequences (Online Resource 4). These results suggest the involvement of OsHRZs and IDEF1 regulators in both the promotive and repressive pathways of JA responses. 


\section{Several genes involved in JA responses are rapidly induced at the onset of Fe deficiency}

To gain further insight into the relationship between JAand $\mathrm{Fe}$ deficiency responses, we investigated the transcriptional responses of the genes involved in JA biosynthesis and signaling in response to early and subsequent $\mathrm{Fe}$ deficiency, as well as HRZ-knockdown (Fig. 3a). Ten out of those 35 genes were induced very rapidly at $3 \mathrm{~h}$ after the onset of Fe deficiency. This induction was sustained for $24 \mathrm{~h}$ and then weakened or vanished at $36 \mathrm{~h}$ and 7 days (Fig. 3a). Moderately enhanced expression in HRZknockdown roots compared with non-transformants was also observed for 12 out of those 35 genes under either Fesufficient or Fe-deficient conditions (Fig. 3a). These results suggest that JA biosynthesis is enhanced during the very early stages of $\mathrm{Fe}$ deficiency in non-transformants and constitutively in HRZ-knockdown plants.

Because of the notably rapid induction of some JA response-related genes under early Fe deficiency, we then compared the kinetics of transcriptional expression of these genes with that of genes known to be involved in $\mathrm{Fe}$ deficiency responses (Fig. 3a vs b). The majority of genes responsible for Fe uptake and translocation, such as those for DMA biosynthesis and transporters, were strongly induced at 24, $36 \mathrm{~h}$ and 7 days of Fe deficiency treatment (Fig. 3b), as reported previously (Itai et al. 2013; Kobayashi et al. 2014). However, these genes began to be induced at no earlier than $9 \mathrm{~h}$ (Fig. 3b; Itai et al. 2013), which is in contrast to the markedly earlier induction of JA-
(A)

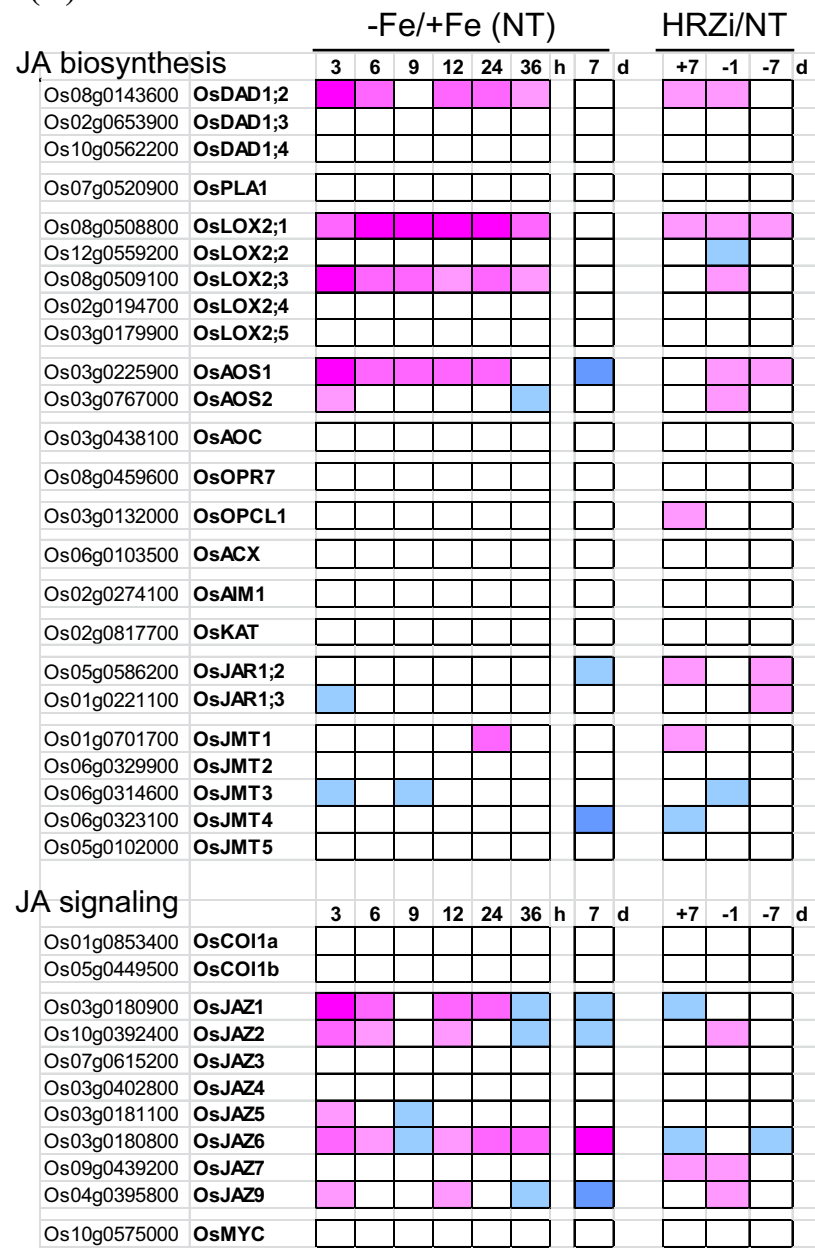

Fig. 3 Transcriptional responses of the genes involved in JA and $\mathrm{Fe}$ deficiency responses in response to Fe deficiency treatment and HRZknockdown in roots. a Genes involved in JA biosynthesis and signaling. b Genes involved in Fe deficiency responses. Expression ratios are shown in the heat maps using a $\log _{2}$ scale based on the microarray results as below. Expression ratios at $3-36 \mathrm{~h}$ and 7 days (d) of Fe deficiency treatment versus control Fe sufficiency
(B)
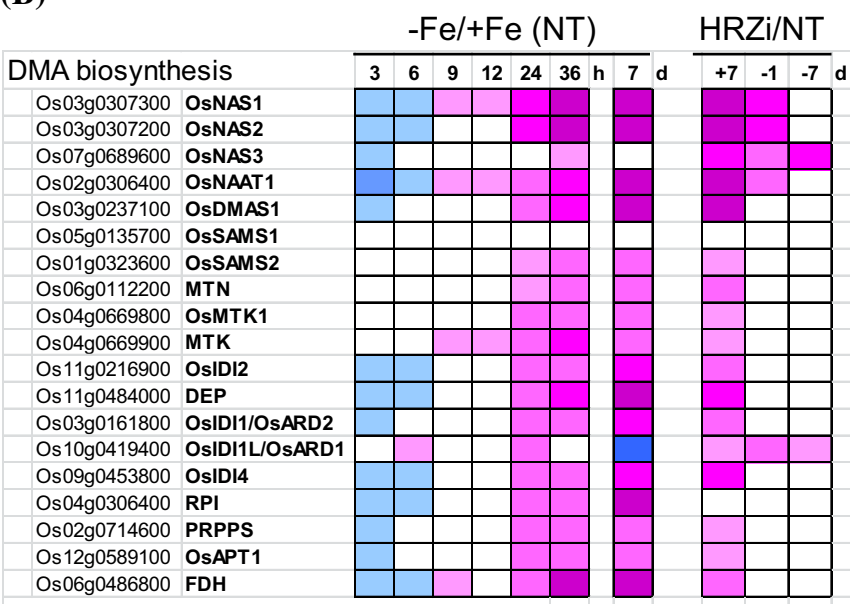

Transporters for Fe uptake/translocation Os1190134900 TOM1 Os02g0650300 OsYSL15 Os03g0667500 OsIRT1 Os03g0667300 OsIRT2 Os07g0258400 OsNRAMP1 Os07g0257200 OsNRAMP5 Os02g0649900 OsYSL2
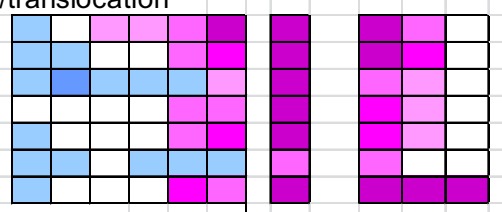

Gene regulation under Fe deficiency Os08g0101000 IDEF1 Os05g0426200 IDEF2 Os01g0952800 OsIRO2 Os01g0689300 OsHRZ1 Os05g0551000 OsHRZ2 Os0190861700 OsHORZ1 Os01g0124200 IBP1.1 Os01g0124400 IBP1.2 Os04g0659300 OsRMC

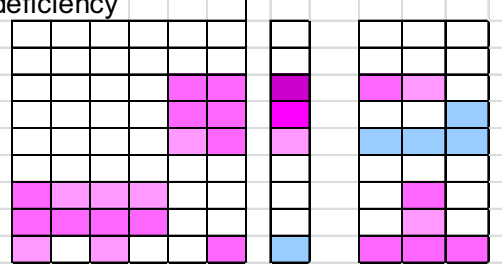

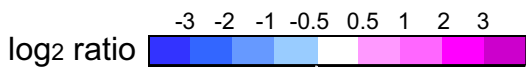

treatment in non-transformants $[-\mathrm{Fe} /+\mathrm{Fe}(\mathrm{NT})]$ are based on previous microarray analyses by Itai et al. (2013) and Ogo et al. (2008), respectively. Expression ratios of HRZ-knockdown versus non-transformants $(\mathrm{HRZi} / \mathrm{NT})$ at day 7 of Fe sufficiency $(+7)$ or day 1 or 7 of deficiency $(-1,-7)$ are based on the previous microarray analysis by Kobayashi et al. (2013) 
Fig. 4 Induction of jasmonates (JAs) during the early stages of Fe deficiency in roots. a Endogenous concentrations of jasmonic acid (JA) and jasmonoyl isoleucine (JA-Ile) (means $\pm \mathrm{SD}, \mathrm{n}=3$ ). b Transcript levels of OsDAD1;2, OsLOX2;1, OsLOX2;3, OsAOS1 and $O s A O S 2$, genes responsible for JA biosynthesis. Non-transgenic (NT) and HRZ-knockdown (HRZi) rice were subjected to $\mathrm{Fe}$ sufficiency $(+\mathrm{Fe})$ or deficiency $(-\mathrm{Fe})$ for 6 and $24 \mathrm{~h}$. Roots were harvested and used for quantification of JAs and quantitative RTPCR. The transcript abundance was normalized against the rice $\alpha-2$ tubulin transcript level and expressed as a ratio relative to the levels in the $\mathrm{NT}+\mathrm{Fe}$ at $6 \mathrm{~h}$ (means $\pm \mathrm{SD}, \mathrm{n}=3$ ). Asterisks indicate significant differences compared with the $+\mathrm{Fe}$ NT level at each time point $(* P<0.05 ; * * P<0.01)$

related genes at $3 \mathrm{~h}$ after the onset of Fe deficiency treatment (Fig. 3a). On the other hand, expression of IBP1.1, $I B P 1.2$ and $O S R M C$ began to be induced at $3 \mathrm{~h}$ (Fig. $3 \mathrm{~b}$ ). These three genes are putative regulators of rice Fe deficiency responses (Yang et al. 2013; Zhang et al. 2014), and their expression is induced by JA treatment (Jiang et al. 2007; Yoshii et al. 2010).

HRZ-knockdown plants hyper-expressed most of the $\mathrm{Fe}$ deficiency-inducible genes involved in $\mathrm{Fe}$ uptake and translocation mostly under Fe sufficiency (Fig. 3b), as reported previously (Kobayashi et al. 2013). Expression of IBP1.1, IBP1.2 and OSRMC genes was also induced in $H R Z$-knockdown plants (Fig. 3b).

\section{JA concentrations in rice roots tend to be increased at the early stages of $\mathrm{Fe}$ deficiency}

The induction of JA biosynthesis-related genes under early Fe deficiency and in HRZ-knockdown plants suggests that the production of JAs is induced in these plants. To prove this hypothesis, we cultured non-transformants and $H R Z$ knockdown plants under Fe deficiency or sufficiency for 6 and $24 \mathrm{~h}$, and quantified endogenous concentrations of JA and JA-Ile, the active form of JA. In non-transgenic roots, concentrations of JA and JA-Ile increased approximately 2-3-fold after Fe deficiency treatment at both 6 and $24 \mathrm{~h}$ (Fig. 4a). Particularly, the JA-Ile concentration was 2.4fold higher in roots subjected to Fe deficiency for $24 \mathrm{~h}$, showing significant increases compared with Fe-sufficiency. In HRZ-knockdown roots, concentrations of JAs, especially JA, were much higher than those in non-transformants under Fe sufficiency (Fig. 4a). Concentrations of JAs under Fe deficiency treatment were similar or moderately lower in HRZ-knockdown roots than in non-transgenic roots. These results strongly suggested that JA biosynthesis is induced during the early stages of Fe deficiency in non-transgenic roots, while this response is derepressed in Fe-sufficient HRZ-knockdown roots.

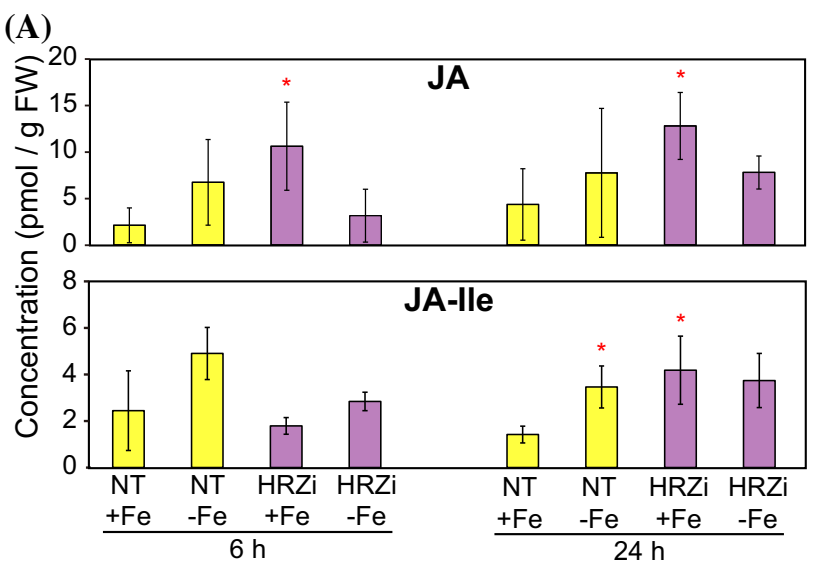

(B)
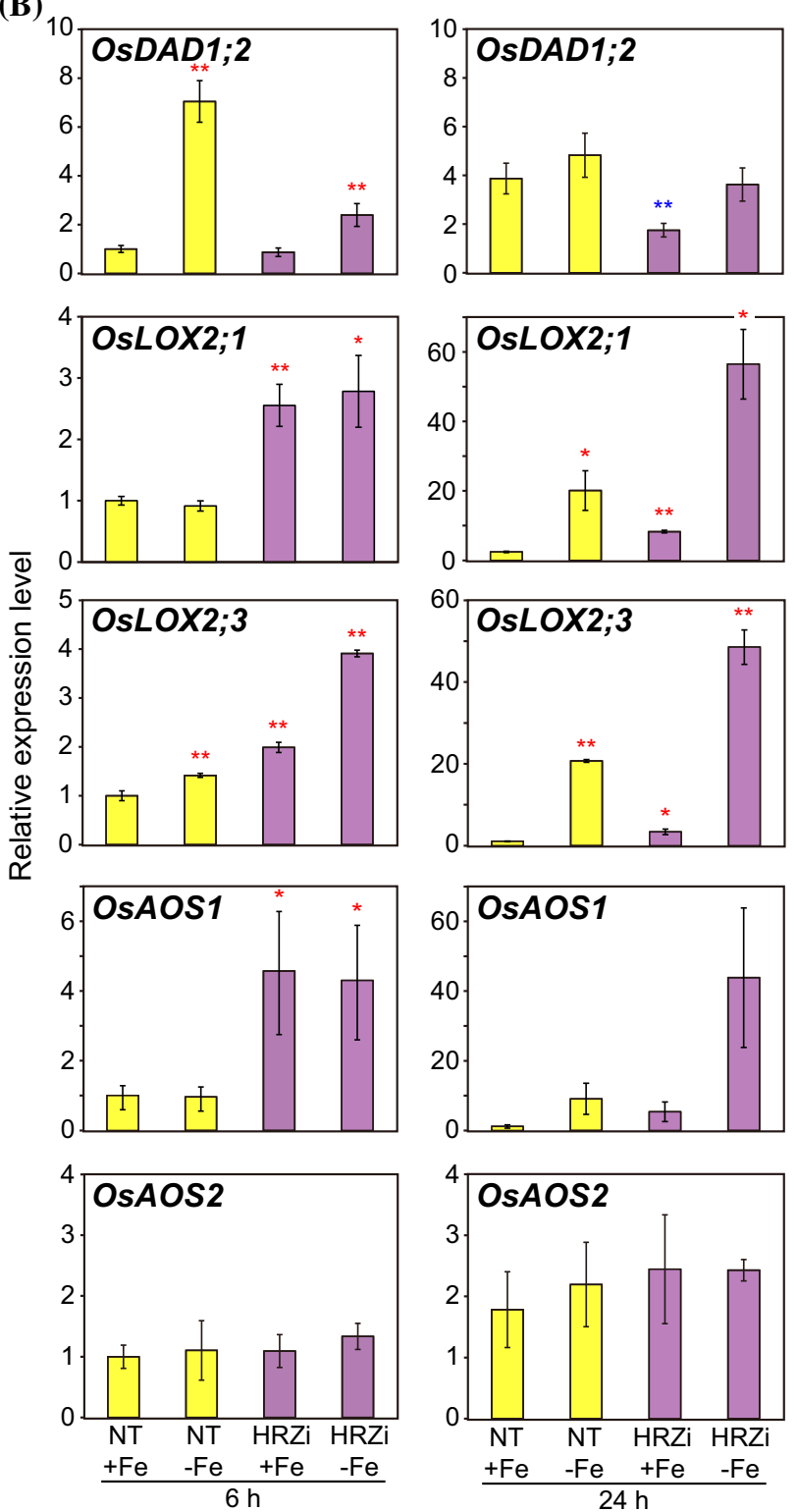
We also confirmed the transcriptional responses of JA biosynthetic genes, OsDAD1;2, OsLOX2;1, OsLOX2;3, $O s A O S 1$ and $O S A O S 2$, in these roots using quantitative RTPCR (Fig. 4b). In non-transgenic roots, OsDAD1;2 and $O s L O X 2 ; 3$ showed induction after $6 \mathrm{~h}$ of Fe deficiency treatment, while OsLOX2;1, OsLOX2;3, and OsAOS1 showed induction after $24 \mathrm{~h}$. OsAOS 2 did not show any induction under Fe deficiency after 6 or $24 \mathrm{~h}$. These transcriptional responses are similar to those observed in previous microarray results, but the induction appears to be slower than the previous results (Fig. 3a). Consistently, expression of OsNAS2, OsIRO2 and OsYSL2, representative $\mathrm{Fe}$ deficiency-inducible genes involved in Fe utilization, were induced only moderately at $24 \mathrm{~h}$ (Online Resource 5), at levels lower than those in the previous microarray results (Fig. 3b; Itai et al. 2013).

The JA biosynthetic genes OsLOX2;1, OsLOX2;3 and $O s A O S 1$, but not $O s D A D 1 ; 2$ and OsAOS2, showed much higher expression in $H R Z$-knockdown plants than in nontransformants under both Fe conditions at both 6 and $24 \mathrm{~h}$ (Fig. 4b). Strong induction was also observed for $O s N A S 2$, OsIRO2 and OsYSL2 (Online Resource 5), consistent with previous results (Fig. 3b; Kobayashi et al. 2013). Repressed expression of $O s H R Z 2$ in $H R Z$-knockdown roots was also confirmed (Online Resource 5). These results confirmed that OsHRZs negatively regulate the expression of some genes involved in the JA signaling pathway, in addition to those involved in Fe utilization.

\section{JAs act positively and negatively on the expression of typical Fe deficiency-inducible genes depending on Fe nutritional status}

We then examined whether expression of genes known to be involved in Fe deficiency responses are transcriptionally regulated by JA (Fig. 5). These genes tended to show moderate and transient induction by JA treatment after 0.5-1 h. Subsequently, a majority of these genes showed strong repression at 3-6 h. This repression was particularly dominant among the genes strongly induced under $\mathrm{Fe}$ deficiency, such as OsNAS1, OsNAS2, OsDMAS1, and TOM1. In contrast, some genes that were not strongly induced under Fe deficiency-including OsSAMS1, OsIDIIL/OsARDI and IDEFI-showed gradual induction by JA treatment after 3-6 h. IBP1.2 and OsRMC showed stronger induction by JA treatment (Fig. 5), consistent with previous reports (Jiang et al. 2007; Yoshii et al. 2010).

We further investigated the effect of endogenous JAs on Fe deficiency responses. For this purpose, we utilized the homozygous cpm2 mutant, in which concentration of endogenous JAs is extremely low because of a mutation in the $O S A O C$ gene, encoding a key enzyme in JAs biosynthesis (Riemann et al. 2013). We measured the transcript

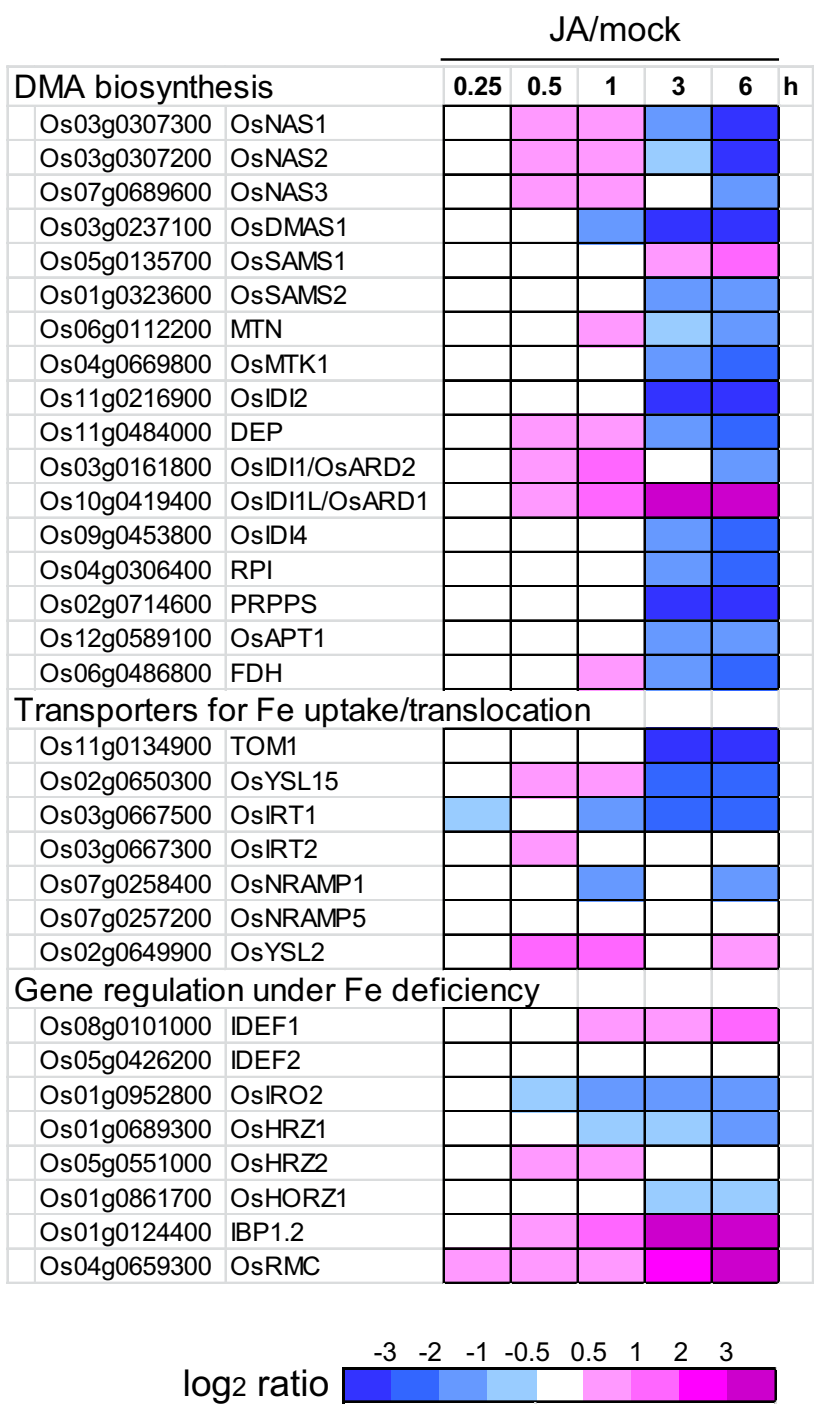

Fig. 5 Transcriptional responses of the genes involved in $\mathrm{Fe}$ deficiency responses to jasmonic acid (JA) treatment in roots. Expression ratios of $100 \mu \mathrm{M} \mathrm{JA}$ treatment versus mock treatment (JA/mock) at $0.25,0.5,1,3$, and $6 \mathrm{~h}$ are based on the microarray results provided on the RiceXPro website (Sato et al. 2013; http:// ricexpro.dna.affrc.go.jp/index.html). The ratios are shown in the heat maps using a $\log _{2}$ scale

levels of representative genes involved in $\mathrm{Fe}$ deficiency responses in wild-type and the cpm 2 mutants cultured under Fe deficiency or sufficiency for $24 \mathrm{~h}$ (Fig. 6). Under $\mathrm{Fe}$ sufficiency, three $\mathrm{Fe}$ deficiency-inducible genes, OsNAS1, OsDMAS1 and OsIRO2, showed higher expression in the cpm2 mutants than in wild-type (Fig. 6a), indicative of negative regulation of these genes by the JA signaling. However, this regulation was diminished after $24 \mathrm{~h}$ of Fe deficiency, except for OsIRO2 (Fig. 6b). Three other Fe deficiency-inducible genes, OsYSL15, OsIRT1 and OsYSL2, showed similar level of expression in wild-type and the cpm2 mutants under both Fe conditions (Fig. 6a, b). Expression level of $I D E F 1$ was higher in the cpm2 
Fig. 6 Transcript levels of representative genes involved in Fe deficiency responses in the JA-deficient cpm 2 mutant. Wild-type (WT) and homozygous cpm 2 mutant were subjected to Fe sufficiency (a) or deficiency (b) for $24 \mathrm{~h}$. Roots were harvested and used for quantitative RT-PCR analysis. The transcript abundance was normalized against the rice $\alpha-2$ tubulin transcript level and expressed as a ratio relative to the levels in the Fe-sufficient WT plants (means $\pm \mathrm{SD}, \mathrm{n}=3$ ). Asterisks indicate significant differences compared with the WT level at each condition $(* P<0.05$; $* * P<0.01)$
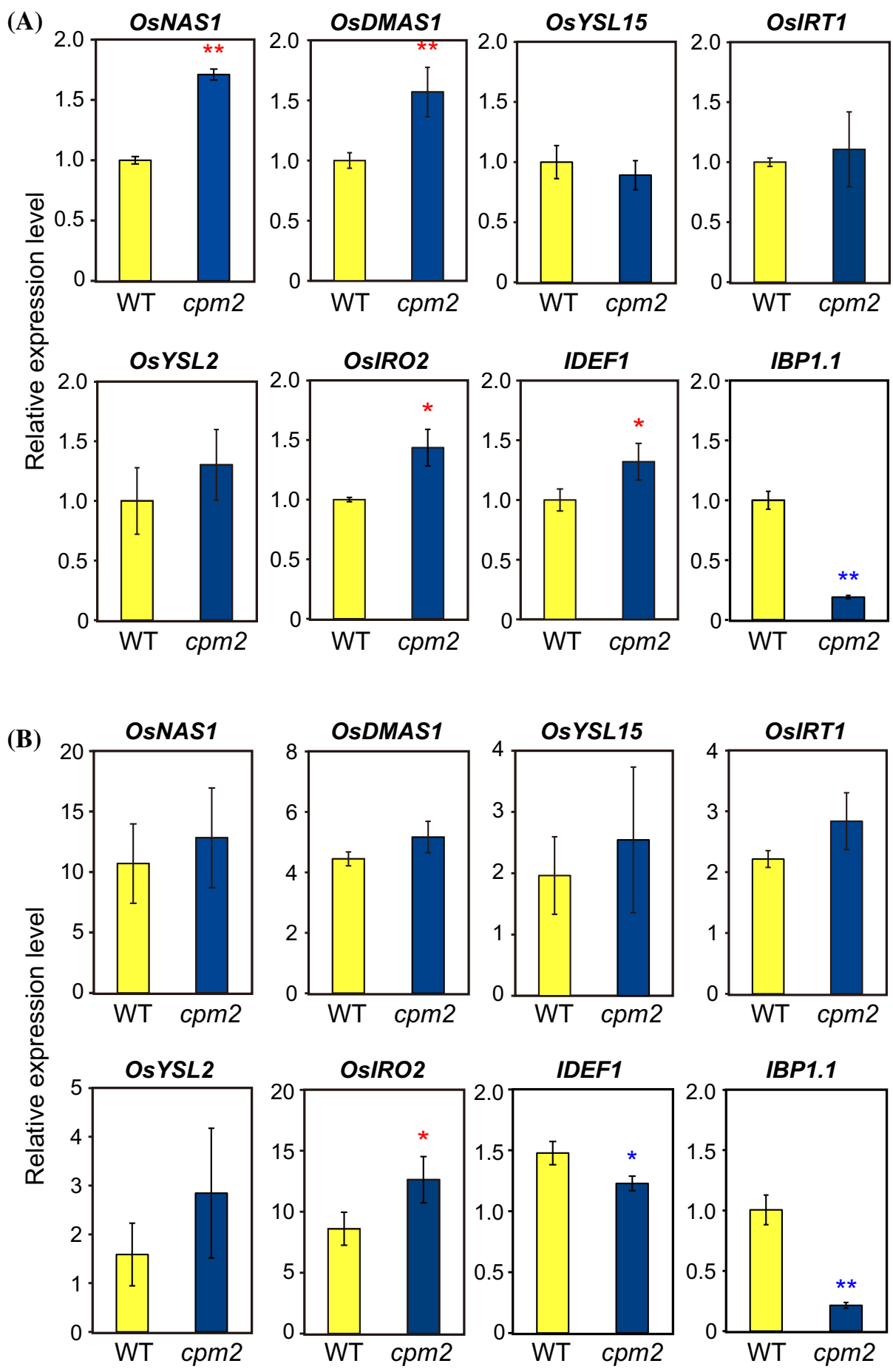

mutants under Fe sufficiency, but was lower under Fe deficiency, compared to wild-type (Fig. 6a, b). Expression of $I B P 1.1$ was strongly repressed in the cpm2 mutants in both Fe conditions (Fig. 6a, b), consistent with the JAinducible nature of this gene (Yoshii et al. 2010). We also confirmed similar repression in expression of OsLOX2;1 and $O s A O S 2$, JA biosynthetic genes induced by JA treatment, in the cpm2 mutants (Online Resource 6). These results indicate that JAs repress a subset of Fe deficiency- inducible genes under Fe-sufficient conditions, but this repression is partly canceled at an early stage of $\mathrm{Fe}$ deficiency.

We also investigated tolerance of the cpm2 mutant to $\mathrm{Fe}$ deficiency. During hydroponic culture under Fe-deficient condition, the cpm 2 mutant showed similar but slightly lower chlorophyll level of the newest leaves, an indicator of Fe deficiency symptoms, compared to wild-type counterpart (Online Resource 7). This result confirmed that 
enhanced expression of some genes involved in Fe uptake and translocation in Fe-sufficient cpm2 mutant does not result in enhanced tolerance to $\mathrm{Fe}$ deficiency.

\section{Discussion}

Involvement of JAs in Fe deficiency responses has been reported only in Arabidopsis, in which administration of methyl-JA exerts negative effects on the expression of the typical Fe deficiency-induced genes IRT1, FRO2 and FIT (Maurer et al. 2011). This report suggested a generally repressive role for $\mathrm{JAs}$ in $\mathrm{Fe}$ deficiency responses (Hindt and Guerinot 2012). However, we have noted the possibility of positive effects of JAs on Fe deficiency responses, based on the putatively positive regulation of rice $\mathrm{Fe}$ deficiency responses by the JA-induced genes IBP1.1, IBP1.2 and OsRMC (Yang et al. 2013; Zhang et al. 2014). A positive effect of JAs on Fe deficiency responses has also been suggested in maize intercropped with peanut, which induces both Fe deficiency responses and proteinlevel expression of key enzymes involved in JA biosynthesis (Xiong et al. 2013a, b). In the present report, we identified the activation of JA signaling during the early stages of $\mathrm{Fe}$ deficiency responses in rice roots. We also found that JAs repress the expression of some genes involved in Fe deficiency responses under Fe sufficiency, but this repression is partly canceled at early stages of Fe deficiency.

When Fe availability decreases, root cells perceive unidentified Fe deficiency signals and start inducing genes responsible for Fe uptake and translocation. This induction was not obvious before $9 \mathrm{~h}$ from the onset of Fe deficiency (Fig. 3b; Itai et al. 2013). We identified that the same plants induce many genes involved in JA biosynthesis and responses during the earlier stages, no later than $3 \mathrm{~h}$ (Figs. 1, 3a). Precedence of the JA responses and increase in the concentration of JAs in Fe deficiency-treated rice roots were also suggested in another culture (Fig. 4, Online Resource 5), although induction of the JA-related and Fe acquisition-related genes was slower in this culture compared to a previous microarray analysis (Fig. 3; Itai et al. 2013). Presumably, the plants in the present experiment experienced less-severe Fe deficiency due to differences in growth conditions, such as plant size. Nevertheless, our results suggested that rapid production of JAs and activation of the JA signaling pathway precede the induction of typical $\mathrm{Fe}$ deficiency-inducible genes at the onset of $\mathrm{Fe}$ deficiency. Thus, the transient production of JAs and activation of the JA pathway might play important roles during the very early stages of Fe deficiency responses.

This hypothesis is supported by the expressional response of Fe deficiency-inducible genes to JA treatment and endogenous JA deficiency. Many of these genes were transiently induced but subsequently repressed by JA administration (Fig. 5). The JA-deficient cpm2 mutant showed higher expression of $\mathrm{Fe}$ deficiency-inducible genes, OsNAS1, OsDMAS1 and OsIRO2, under Fe sufficiency (Fig. 6a). Thus, these genes are thought to be repressed by JAs, similarly to Arabidopsis Fe deficiencyinducible genes (Maurer et al. 2011), at normal Fe condition. However, this repression was canceled under early $\mathrm{Fe}$ deficiency, except for OsIRO2 (Fig. 6b). These results indicate that the repressive effect of JAs in rice roots is dependent on Fe nutritional status, and is mitigated at early stages of Fe deficiency. This might be due to promotive effect of transiently synthesized JAs at very early stages of Fe deficiency, which might counteract the constitutive and repressive effect of JAs. The JA pathway might fine-tune the expression kinetics of Fe deficiency-induced genes. In Arabidopsis, repression of typical Fe deficiency-induced genes was observed after methyl-JA treatment for $6 \mathrm{~h}$ or 3 days (Maurer et al. 2011). Analysis of earlier responses to JA administration is necessary to confirm whether JA exhibits promotive effects in Arabidopsis and other plant species similar to those in rice.

The involvement of JAs in rice Fe deficiency responses is summarized in Fig. 7. Our results indicate that the effects of JAs are dependent on Fe status, and also on IDEF1 and OsHRZs, which are positive and negative regulators of $\mathrm{Fe}$ deficiency responses, respectively (Kobayashi et al. 2007, 2009, 2013, 2014). Our correlation analysis suggested that JA-responsive genes are positively regulated by IDEF1 at both 1 and 7 days of Fe deficiency (Fig. 2a), which correspond to early and subsequent stages of IDEF1-mediated Fe deficiency responses, respectively (Kobayashi et al. 2009). Because IDEF1 is especially important for early $\mathrm{Fe}$ deficiency responses (Kobayashi et al. 2009), the JA pathway might be involved in triggering these responses in roots. In addition, $I D E F 1$ and its possible activator IBP1.2 showed gradual induction by JA treatment over 1-6 h, in contrast to repression of typical $\mathrm{Fe}$ deficiency-induced genes by JA treatment after 3-6 h (Fig. 5). Although expression of IBP1.1, a close homolog of $I B P 1.2$, is not available in this database, a previous report indicated that expression of IBPI.I (Os01g0124200) and IBP1.2 (Os01g0124400) is similarly induced by JA treatment at 12 days (Yoshii et al. 2010). IBP1.1 and IBP1.2 interact with IDEF1, putatively supporting the function of IDEF1 by preventing its proteasomal degradation (Zhang et al. 2014). Expressional analysis in the cpm 2 mutant also indicated that expression of IDEFI and $I B P 1.1$ is induced by JAs under early $\mathrm{Fe}$ deficiency (Fig. 6b). Under Fe sufficiency, expression of IBP1.1 was similar, but IDEFI expression showed the opposite tendency (Fig. 6a). Taken together, positive interaction 
(A) $+\mathrm{Fe}$

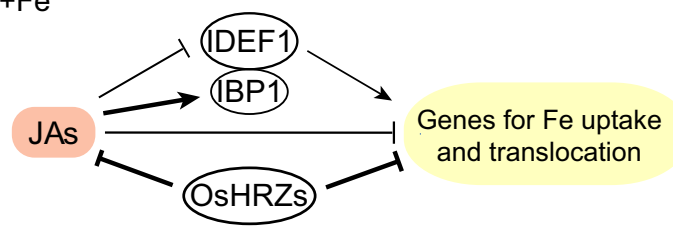

(B) $-\mathrm{Fe}$ (early)

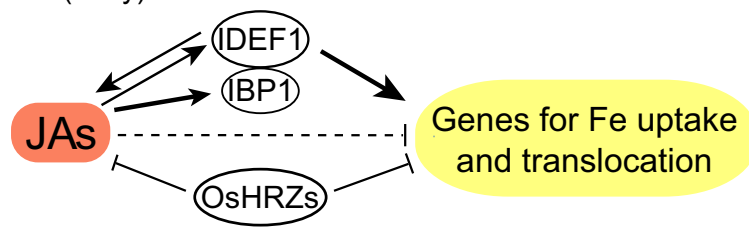

Fig. 7 Summary of the regulation of the genes involved in Fe uptake and translocation by JAs, IDEF1 and OsHRZs in rice roots. a Fesufficient conditions. b Early stages of Fe-deficient conditions. Expressional regulation identified previously (Kobayashi et al. $2009,2013,2014)$ and in the present report is depicted. The thickness of the lines indicates the relative strength of the regulation. The majority of genes responsible for $\mathrm{Fe}$ uptake and translocation are induced in response to Fe deficiency (Itai et al. 2013; Kobayashi et al. 2014). Prior to this induction, concentrations of JA and JA-Ile tend to be increased, which might be responsible for mitigation of constitutive repression of the genes involved in Fe uptake and translocation by JAs (dashed line in (b)). The transcription factor IDEF1 positively regulates major genes involved in Fe uptake and translocation, and this regulation is intensified during early stages of Fe-deficient conditions (Kobayashi et al. 2009). Conversely, the ubiquitin ligase OsHRZs negatively regulate major genes involved in Fe uptake and translocation, and this regulation become less obvious with the progression of Fe deficiency (Kobayashi et al. 2013). IBP1 binds to IDEF1 and is supposed to enhance the stability and function of IDEF1 (Zhang et al. 2014)

between the IDEF1 pathway and JA signaling occurs during the early stages of Fe deficiency (Fig. 7b).

Our results also revealed that OsHRZ ubiquitin ligases suppress the JA pathway, especially under Fe sufficiency (Fig. 7). Expression analysis of HRZ-knockdown lines indicated that OsHRZs negatively regulate the expression of late JA-responsive genes and several genes involved in JA biosynthesis and signaling (Figs. 2a, 3a). In addition, HRZ-knockdown roots accumulated more JAs than did non-transformants under Fe sufficiency (Fig. 4a). OsHRZs and their Arabidopsis homolog BTS are Fe- and Zn-binding ubiquitin ligases that negatively regulate $\mathrm{Fe}$ deficiency responses (Long et al. 2010; Kobayashi et al. 2013; Selote et al. 2015), possibly preventing excessive Fe uptake and translocation. Repression of the JA pathway by OsHRZs revealed in the present study (Figs. 2a, 3a, 4) suggests mitigation of this Fe-limiting function of HRZs/BTS by preventing JA-mediated repression of typical genes.

Although OsHRZs constitutively repress Fe uptake and translocation, as evidenced by the Fe-accumulating phenotype of HRZ-knockdown lines irrespective of Fe nutritional status (Kobayashi et al. 2013), several lines of evidence suggest that HRZs/BTS alter or modify their functions in response to $\mathrm{Fe}$ availability. Expression of HRZs/BTS is transcriptionally induced under Fe deficiency, although their induction levels are somewhat lower than those of typical $\mathrm{Fe}$ deficiency-inducible genes involved in $\mathrm{Fe}$ homeostasis (Fig. 3b; Long et al. 2010; Kobayashi et al. 2013). In addition, BTS protein is destabilized under Fe-rich conditions in vitro (Selote et al. 2015), suggesting preferential roles for HRZs/BTS under Fe-deficient conditions. In contrast to these observations, the transcriptional profiles of HRZknockdown rice lines indicated that OsHRZs repress the expression of major genes involved in $\mathrm{Fe}$ uptake and translocation preferentially under Fe-sufficient conditions (Fig. 3b; Kobayashi et al. 2013). In the present study, we found higher concentrations of JAs in HRZ-knockdown lines compared with non-transformants only under Fe sufficiency (Fig. 4a), although the transcript levels of OsLOX2;1, $O s L O X 2 ; 3$ and OsAOS1, genes responsible for JA biosynthesis, were markedly higher in HRZ-knockdown lines under both Fe-sufficient and -deficient conditions (Fig. 4b). This phenomenon might be due to failure of further induction of rate-limiting enzyme(s) for JA biosynthesis in HRZ-knockdown lines, or a feedback mechanism for limiting JA concentrations under Fe deficiency. The preferential effect of OsHRZs on JA concentrations under Fe-sufficient conditions coincides with their predominant regulation of $\mathrm{Fe}$ acquisition-related genes under Fe sufficiency (Fig. 3b; Kobayashi et al. 2013). Interestingly, accumulation of endogenous JA-Ile was less dominant than that of JA in Fesufficient HRZ-knockdown roots (Fig. 4a), although the expression of OSJARI; 2, encoding a putative enzyme for JAIle biosynthesis from JA (Hirano et al. 2008), was enhanced in Fe-sufficient HRZ-knockdown roots (Fig. 3a). Possibly, protein degradation or inactivation of OsJAR $1 ; 2$ or other biosynthetic genes for JA-Ile might occur in Fe-sufficient $H R Z$-knockdown roots to prevent excessive JA signaling.

The increased concentrations of JAs in HRZ-knockdown plants under normal $\mathrm{Fe}$ supply also suggest altered responses of these plants to various biotic and abiotic stresses regulated by the JA pathways (Fonseca et al. 2009; Vleesschauwer et al. 2013). Indeed, close homologs of HRZs/BTS in Lotus japonicus and tobacco regulate the responses to bacterial and viral infections, respectively (Shimomura et al. 2006; Yamaji et al. 2010). HRZs/BTS and their homologs are widely distributed in the plant kingdom (Urzica et al. 2012; Kobayashi et al. 2013), and might play significant roles linking Fe deficiency responses, JA responses and other stress responses in plants.

In conclusion, we clarified that JA biosynthesis and signaling pathways are rapidly induced in rice roots in response to very early Fe deficiency, and are constitutively activated by $H R Z$-knockdown. JAs negatively regulate some $\mathrm{Fe}$ deficiency-inducible genes under $\mathrm{Fe}$-sufficient 
conditions, but this regulation is partly canceled under early Fe deficiency. The JA signaling in early Fe deficiency responses is partly regulated by IDEF1 and OsHRZs. Identification of the molecular mechanisms by which IDEF1 and OsHRZs sense and transmit Fe deficiency signaling will be key to clarifying the corresponding signaling pathways.

Acknowledgments We thank Dr. Moritoshi lino (Osaka City University, Japan), Dr. Hisakazu Yamane, Dr. Koji Miyamoto (Teikyo University, Japan) and Dr. Kazunori Okada (The University of Tokyo, Japan) for providing us with the cpm2 mutant. We also thank Dr. Haruhiko Inoue (National Institute of Agrobiological Sciences, Japan) for valuable discussion on JA responses and Ms. May Linn Aung and Ms. Kazuko Arai (Ishikawa Prefectural University, Japan) for assistance with rice culture and analysis. This research was supported by the Japan Science and Technology Agency program PRESTO (to T. K.) and in part by the Japan Science and Technology Agency program ALCA (to N. K. N.), and JSPS KAKENHI Grant Numbers $15 \mathrm{H} 01187$ and $15 \mathrm{H} 05617$ (to T. K.).

Author contribution T.K. and N.K.N. designed the research. T.K. carried out most of the experiments with assistance from R.N.I., T.S., and H.N. T.O. carried out JA quantification with assistance from Y.I. and M.U. T.K. analyzed the data. T.K. wrote the manuscript with contributions and discussion from all of the co-authors.

Open Access This article is distributed under the terms of the Creative Commons Attribution 4.0 International License (http://crea tivecommons.org/licenses/by/4.0/), which permits unrestricted use, distribution, and reproduction in any medium, provided you give appropriate credit to the original author(s) and the source, provide a link to the Creative Commons license, and indicate if changes were made.

\section{References}

Armengaud P, Breitling R, Amtmann A (2004) The potassiumdependent transcriptome of Arabidopsis reveals a prominent role of jasmonic acid in nutrient signaling. Plant Physiol 136:2556-2576

Armengaud P, Breitling R, Amtmann A (2010) Coronatine-insensitive 1 (COI1) mediates transcriptional responses of Arabidopsis thaliana to external potassium supply. Mol Plant 3:390-405

Barberon M, Zelazny E, Robert S, Conéjéro G, Curie C, Friml J, Vert G (2011) Monoubiquitin-dependent endocytosis of the IRONREGULATED TRANSPORTER 1 (IRT1) transporter controls iron uptake in plants. Proc Natl Acad Sci USA 108:E450-E458

Colangelo EP, Guerinot ML (2004) The essential basic helix-loophelix protein FIT1 is required for the iron deficiency response. Plant Cell 16:3400-3412

Curie C, Panaviene Z, Loulergue C, Dellaporta SL, Briat JF, Walker EL (2001) Maize yellow stripe 1 encodes a membrane protein directly involved in Fe(III) uptake. Nature 409:346-349

Curie C, Cassin G, Couch D, Divol F, Higuchi K, Le Jean M, Misson J, Schikora A, Czernic P, Mari S (2009) Metal movement within the plant: contribution of nicotianamine and yellow stripe 1-like transporters. Ann Bot 103:1-11

Eide D, Broderius M, Fett J, Guerinot ML (1996) A novel ironregulated metal transporter from plants identified by functional expression in yeast. Proc Natl Acad Sci USA 93:5624-5628
Fonseca S, Chico JM, Solano R (2009) The jasmonate pathway: the ligand, the receptor and the core signaling module. Curr Opin Plant Biol 12:539-547

Fourcroy P, Sisó-Terraza P, Sudre D, Savirón M, Reyt G, Gaymard F, Abadía A, Abadía J, Álvarez-Fernández A, Briat JF (2014) Involvement of the ABCG37 transporter in secretion of scopoletin and derivatives by Arabidopsis roots in response to iron deficiency. New Phytol 201:155-167

García MJ, Suárez V, Romera FJ, Alcántara E, Pérez-Vicente R (2011) A new model involving ethylene, nitric oxide and Fe to explain the regulation of Fe-acquisition genes in Strategy I plants. Plant Physiol Biochem 49:537-544

Hindt MN, Guerinot ML (2012) Getting a sense for signals: regulation of the plant iron deficiency response. Biochim Biophys Acta 1823:1521-1530

Hirano K, Aya K, Hobo T, Sakakibara H, Kojima M, Shim RA, Hasegawa Y, Ueguchi-Tanaka M, Matsuoka M (2008) Comprehensive transcriptome analysis of phytohormone biosynthesis and signaling genes in microspore/pollen and tapetum of rice. Plant Cell Physiol 49:1429-1450

Inoue H, Kobayashi T, Nozoye T, Takahashi M, Kakei Y, Suzuki K, Nakazono M, Nakanishi H, Mori S, Nishizawa NK (2009) Rice OsYSL15 is an iron-regulated iron(III)-deoxymugineic acid transporter expressed in the roots and is essential for iron uptake in early growth of the seedlings. J Biol Chem 284:3470-3479

Ishimaru Y, Suzuki M, Tsukamoto T, Suzuki K, Nakazono M, Kobayashi T, Wada Y, Watanabe S, Matsuhashi S, Takahashi M, Nakanishi H, Mori S, Nishizawa NK (2006) Rice plants take up iron as an $\mathrm{Fe}^{3+}$-phytosiderophore and as $\mathrm{Fe}^{2+}$. Plant $\mathbf{J}$ 45:335-346

Itai RN, Ogo Y, Kobayashi T, Nakanishi H, Nishizawa NK (2013) Rice genes involved in phytosiderophore biosynthesis are synchronously regulated during the early stages of iron deficiency in roots. Rice 6:16

Jakoby M, Wang HY, Reidt W, Weisshaar B, Bauer P (2004) FRU (BHLH029) is required for induction of iron mobilization genes in Arabidopsis thaliana. FEBS Lett 577:528-534

Jiang J, Li J, Xu Y, Han Y, Bai Y, Zhou G, Lou Y, Xu Z, Chong K (2007) RNAi knockdown of Oryza sativa root meander curling gene led to altered root development and coiling which were mediated by jasmonic acid signaling in rice. Plant Cell Environ 30:690-699

Kakei Y, Ogo Y, Itai RN, Kobayashi T, Yamakawa T, Nakanishi H, Nishizawa NK (2013) Development of a novel prediction method of cis-elements to hypothesize collaborative functions of cis-element pairs in iron-deficient rice. Rice 6:22

Kobayashi T, Nishizawa NK (2012) Iron uptake, translocation, and regulation in higher plants. Annu Rev Plant Biol 63:131-152

Kobayashi T, Ogo Y, Itai RN, Nakanishi H, Takahashi M, Mori S, Nishizawa NK (2007) The transcription factor IDEF1 regulates the response to and tolerance of iron deficiency in plants. Proc Natl Acad Sci USA 104:19150-19155

Kobayashi T, Itai RN, Ogo Y, Kakei Y, Nakanishi H, Takahashi M, Nishizawa NK (2009) The rice transcription factor IDEF1 is essential for the early response to iron deficiency, and induces vegetative expression of late embryogenesis abundant genes. Plant J 60:948-961

Kobayashi T, Itai RN, Aung MS, Senoura T, Nakanishi H, Nishizawa NK (2012) The rice transcription factor IDEF1 directly binds to iron and other divalent metals for sensing cellular iron status. Plant J 69:81-91

Kobayashi T, Nagasaka S, Senoura T, Itai RN, Nakanishi H, Nishizawa NK (2013) Iron-binding haemerythrin RING ubiquitin ligases regulate plant iron responses and accumulation. Nat Commun 4:2792 
Kobayashi T, Itai RN, Nishizawa NK (2014) Iron deficiency responses in rice roots. Rice 7:27

Lan P, Li W, Wen TN, Shiau JY, Wu YC, Lin W, Schmidt W (2011) iTRAQ protein profile analysis of Arabidopsis roots reveals new aspects critical for iron homeostasis. Plant Physiol 155:821-834

Lei GJ, Zhu XF, Wang ZW, Dong F, Dong NY, Zheng SJ (2014) Abscisic acid alleviates iron deficiency by promoting root iron reutilization and transport from root to shoot in Arabidopsis. Plant Cell Environ 37:852-863

Lingam P, Mohrbacher J, Brumbarova T, Potuschak T, Fink-Straube C, Blondet E, Genschik P, Bauer P (2011) Interaction between the bHLH transcription factor FIT and ETHYLENE INSENSITIVE3/ETHYLENE INSENSITIVE3-LIKE1 reveals molecular linkage between the regulation of iron acquisition and ethylene signaling in Arabidopsis. Plant Cell 23:1815-1829

Long TA, Tsukagoshi H, Busch W, Lahner B, Salt D, Benfey PN (2010) The bHLH transcription factor POPEYE regulates response to iron deficiency in Arabidopsis roots. Plant Cell 22:2219-2236

Matsuoka K, Furukawa J, Bidadi H, Asahina M, Yamaguchi S, Satoh S (2014) Gibberellin-induced expression of Fe uptake-related genes in Arabidopsis. Plant Cell Physiol 55:87-98

Maurer F, Müller S, Bauer P (2011) Suppression of Fe deficiency gene expression by jasmonate. Plant Physiol Biochem 49:530-536

Murata Y, Ma JF, Yamaji N, Ueno D, Nomoto K, Iwashita T (2006) A specific transporter for iron(III)-phytosiderophore in barley roots. Plant J 46:563-572

Nozoye T, Nagasaka S, Kobayashi T, Takahashi M, Sato Y, Sato Y, Uozumi N, Nakanishi H, Nishizawa NK (2011) Phytosiderophore efflux transporters are crucial for iron acquisition in graminaceous plants. J Biol Chem 286:5446-5454

Ogo Y, Itai RN, Nakanishi H, Inoue H, Kobayashi T, Suzuki M, Takahashi M, Mori S, Nishizawa NK (2006) Isolation and characterization of IRO2, a novel iron-regulated bHLH transcription factor in graminaceous plants. J Exp Bot 57:2867-2878

Ogo Y, Itai RN, Nakanishi H, Kobayashi T, Takahashi M, Mori S, Nishizawa NK (2007) The rice bHLH protein OsIRO2 is an essential regulator of the genes involved in $\mathrm{Fe}$ uptake under $\mathrm{Fe}$ deficient conditions. Plant J 51:366-377

Ogo Y, Kobayashi T, Itai RN, Nakanishi H, Kakei Y, Takahashi M, Toki S, Mori S, Nishizawa NK (2008) A novel NAC transcription factor IDEF2 that recognizes the iron deficiency-responsive element 2 regulates the genes involved in iron homeostasis in plants. J Biol Chem 283:13407-13417

Ogo Y, Itai RN, Kobayashi T, Aung MS, Nakanishi H, Nishizawa NK (2011) OsIRO2 is responsible for iron utilization in rice and improves growth and yield in calcareous soil. Plant Mol Biol 75:593-605

Palmer CM, Hindt MN, Schmidt H, Clemens S, Guerinot ML (2013) MYB10 and MYB72 are required for growth under iron-limiting conditions. PLoS Genet 9:e1003953

Riemann M, Haga K, Shimizu T, Okada K, Ando S, Mochizuki S, Nishizawa Y, Yamanouchi U, Nick P, Yano M, Minami E, Takano M, Yamane H, Iino M (2013) Identification of rice Allene Oxide Cyclase mutants and the function of jasmonate for defence against Magnaporthe oryzae. Plant J 74:226-238

Robinson NJ, Procter CM, Connolly EL, Guerinot ML (1999) A ferric-chelate reductase for iron uptake from soils. Nature 397:694-697

Rodríguez-Celma J, Lin WD, Fu GM, Abadía J, López-Millán AF, Schmidt W (2013) Mutually exclusive alterations in secondary metabolism are critical for the uptake of insoluble iron compounds by Arabidopsis and Medicago truncatula. Plant Physiol 162:1473-1485

Römheld V, Marschner H (1986) Evidence for a specific uptake system for iron phytosiderophore in roots of grasses. Plant Physiol 80:175-180
Santi S, Schmidt W (2009) Dissecting iron deficiency-induced proton exclusion in Arabidopsis roots. New Phytol 183:1072-1084

Sato C, Seto Y, Nabeta K, Matsuura H (2009) Kinetics of the accumulation of jasmonic acid and its derivatives in systemic leaves of tobacco (Nicotiana tabacum cv. Xanthi nc) and translocation of deuterium-labeled jasmonic acid from the wounding site to the systemic site. Biosci Biotech Biochem 73:1962-1970

Sato Y, Takehisa H, Kamatsuki K, Minami H, Namiki N, Ikawa H, Ohyanagi H, Sugimoto K, Antonio B, Nagamura Y (2013) RiceXPro Version 3.0: expanding the informatics resource for rice transcriptome. Nucleic Acids Res 41:D1206-D1213

Schmid NB, Giehl RFH, Döll S, Mock HP, Strehmel N, Scheel D, Kong X, Hider RC, von Wirén N (2014) Feruloyl-CoA 6'hydroxylase1-dependent coumarins mediate iron acquisition from alkaline substrates in Arabidopsis. Plant Physiol 164:160-172

Schmidt W, Buckhout TJ (2011) A hitchhiker's guide to the Arabidopsis ferrome. Plant Physiol Biochem 49:462-470

Séguéla M, Briat JF, Vert G, Curie C (2008) Cytokinins negatively regulate the root iron uptake machinery in Arabidopsis through a growth-dependent pathway. Plant J. 55:289-300

Selote D, Samira R, Matthiadis A, Gillikin JW, Long TA (2015) Ironbinding E3 ligase mediates iron response in plants by targeting bHLH transcription factors. Plant Physiol 167:273-286

Seo JS, Joo J, Kim MJ, Kim YK, Nahm BH, Song SI, Cheong JJ, Lee JS, Kim JK, Choi YD (2011) OsbHLH148, a basic helix-loophelix protein, interacts with OsJAZ proteins in a jasmonate signaling pathway leading to drought tolerance in rice. Plant $\mathrm{J}$ 65:907-921

Shimomura K, Nomura M, Tajima S, Kouchi H (2006) LjnsRING, a novel RING finger protein, is required for symbiotic interactions between Mesorhizobium loti and Lotus japonicus. Plant Cell Physiol 47:1572-1581

Shin LJ, Lo JC, Chen GH, Callis J, Fu H, Yeh KC (2013) IRT1 degradation factor1, a ring E3 ubiquitin ligase, regulates the degradation of iron-regulated transporter1 in Arabidopsis. Plant Cell 25:3039-3051

Sivitz A, Grinvalds C, Barberon M, Curie C, Vert G (2011) Proteasome-mediated turnover of the transcriptional activator FIT is required for plant iron-deficiency responses. Plant $\mathbf{J}$ 66:1044-1052

Sivitz AB, Hermand V, Curie C, Vert G (2012) Arabidopsis bHLH100 and bHLH101 control iron homeostasis via a FITindependent pathway. PLoS One 7:e44843

Takagi S (1976) Naturally occurring iron-chelating compounds in oatand rice-root washing. I. Activity measurement and preliminary characterization. Soil Sci Plant Nutr 22:423-433

Takehisa H, Sato Y, Antonio BA, Nagamura Y (2013) Global transcriptome profile of rice root in response to essential macronutrient deficiency. Plant Signal Behav 8:e24409

Urzica EI, Casero D, Yamasaki H, Hsieh SI, Adler LN, Karpowicz SJ, Blaby-Haas CE, Clarke SG, Loo JA, Pellegrini M, Merchant SS (2012) Systems and trans-system level analysis identifies conserved iron deficiency responses in the plant lineage. Plant Cell 24:3921-3948

Vert G, Grotz N, Dédaldéchamp F, Gaymard F, Guerinot ML, Briat JF, Curie C (2002) IRT1, an Arabidopsis transporter essential for iron uptake from the soil and for plant growth. Plant Cell 14:1223-1233

Vleesschauwer DD, Gheysen G, Höfte M (2013) Hormone defense networking in rice: tales from a different world. Trends Plant Sci 18:555-565

Wang B, Li Y, Zhang WH (2012) Brassinosteroids are involved in response of cucumber (Cucumis sativus) to iron deficiency. Ann Bot 110:681-688 
Wang L, Ying Y, Narsai R, Ye L, Zheng L, Tian J, Whelan J, Shou H (2013a) Identification of OsbHLH133 as a regulator of iron distribution between roots and shoots in Oryza sativa. Plant Cell Environ 36:224-236

Wang N, Cui Y, Liu Y, Fan H, Du J, Huang Z, Yuan Y, Wu H, Ling HQ (2013b) Requirement and functional redundancy of $\mathrm{Ib}$ subgroup bHLH proteins for iron deficiency responses and uptake in Arabidopsis thaliana. Mol Plant 6:503-513

Wang B, Li G, Zhang WH (2015) Brassinosteroids are involved in Fe homeostasis in rice (Oryza sativa L.). J Exp Bot 66:2749-2761

Xiong H, Kakei Y, Kobayashi T, Guo X, Nakazono M, Takahashi H, Nakanishi H, Shen H, Zhang F, Nishizawa NK, Zuo Y (2013a) Molecular evidence for phytosiderophore-induced improvement of iron nutrition of peanut intercropped with maize in calcareous soil. Plant Cell Environ 36:1888-1902

Xiong H, Shen H, Zhang L, Zhang Y, Guo X, Wang P, Duan P, Ji C, Zhong L, Zhang F, Zuo Y (2013b) Comparative proteomic analysis for assessment of the ecological significance of maize and peanut intercropping. J Proteomics 78:447-460

Yamaji Y, Hamada K, Yoshinuma T, Sakurai K, Yoshii A, Shimizu T, Hashimoto M, Suzuki M, Namba S, Hibi T (2010) Inhibitory effect on the tobacco mosaic virus infection by a plant RING finger protein. Virus Res 153:50-57

Yang DL, Yao J, Mei CS, Tong XH, Zeng LJ, Li Q, Xiao LT, Sun TP, Li J, Deng XW, Lee CM, Thomashow MF, Yang Y, He Z, He SY (2012) Plant hormone jasmonate prioritizes defense over growth by interfering with gibberellin signaling cascade. Proc Natl Acad Sci USA 109:E1192-E1200
Yang A, Li Y, Xu Y, Zhang WH (2013) A receptor-like protein RMC is involved in regulation of iron acquisition in rice. J Exp Bot 64:5009-5020

Yoshii M, Yamazaki M, Rakwal R, Kishi-Kaboshi M, Miyao A, Hirochika H (2010) The NAC transcription factor RIM1 of rice is a new regulator of jasmonate signaling. Plant $\mathrm{J}$ 61:804-815

Yuan YX, Zhang J, Wang DW, Ling HQ (2005) AtbHLH29 of Arabidopsis thaliana is a functional ortholog of tomato FER involved in controlling iron acquisition in strategy I plants. Cell Res 15:613-621

Yuan Y, Wu H, Wang N, Li J, Zhao W, Du J, Wang D, Ling HQ (2008) FIT interacts with AtbHLH38 and AtbHLH39 in regulating iron uptake gene expression for iron homeostasis in Arabidopsis. Cell Res 18:385-397

Zhang L, Itai RN, Yamakawa $T$, Nakanishi $H$, Nishizawa NK, Kobayashi T (2014) The Bowman-Birk trypsin inhibitor IBP1 interacts with and prevents degradation of IDEF1 in rice. Plant Mol Biol Rep 32:841-851

Zhang J, Liu B, Li M, Feng D, Jin H, Wang P, Liu J, Xiong F, Wang J, Wang HB (2015) The bHLH transcription factor bHLH104 interacts with IAA-LEUCINE RESISTANT3 and modulates iron homeostasis in Arabidopsis. Plant Cell 27:787-805

Zheng L, Ying Y, Wang L, Wang F, Whelan J, Shou H (2010) Identification of a novel iron regulated basic helix-loop-helix protein involved in Fe homeostasis in Oryza sativa. BMC Plant Biol 10:166 